\title{
Disponibilidade e uso de recursos alóctones por Bryconamericus microcephalus (Miranda-Ribeiro) (Actinopterygii, Characidae), no córrego Andorinha, Ilha Grande, Rio de Janeiro, Brasil
}

\author{
Carla Ferreira Rezende \& Rosana Mazzoni
}

Departamento de Ecologia Laboratório de Ecologia de Peixes, Universidade do Estado do Rio de Janeiro. Avenida São Francisco Xavier 24, 20550-013 Rio de Janeiro, Rio de Janeiro, Brasil. E-mail: carla.fr@terra.com.br; mazzoni@uerj.br

\begin{abstract}
Availability and use of allochthonous resources for Bryconamericus microcephalus (Miranda-Ribeiro) (Actinopterygii, Characidae), at Andorinha Stream, Ilha Grande, Rio de Janeiro, Brazil. The present work was conducted at Andorinha stream, oriental slope of Ilha Grande, and aimed the analysis about the correlation between allochthonous preys input and the use of such preys as food resource for Bryconamericus microcephalus (Miranda-Ribeiro, 1908). Quantification of imported preys was done during May/2002 and February/2003 together with fish samples used for diet analysis. Feeding selectivity was established for Young and adult individuals according to prey size and taxonomical order. Ivlev Electivity Index (IEI) was applied for the abundance of consumed preys and their environmental availability. Homoptera (IEI $=0,94)$, Coleoptera (IEI $=0,57$ ) and Diptera $(\mathrm{IEI}=0,50)$ were preferred among adult individuals while Hemiptera $(0,98)$, Diptera $(0,60)$ and Homoptera $(0,98)$ preferred among young individuals. Despite their high environmental availability Hymenoptera (IEI = 0,$15 ;-0,42$ ) and Orthoptera (IEI $=-0,52 ;-1$ ) were rejected among adult and young individuals, respectively, suggesting that they were eaten because their abundance. These results indicated that young and adult individuals of $B$. microcephalus presented quite different feeding preferences.
\end{abstract}

KEY WORDS. Diet; selectivity; Rain Forest.

RESUMO. O presente estudo foi desenvolvido no córrego Andorinha, localizado na vertente oriental da Illha Grande, e teve como objetivo relacionar a disponibilidade de presas alóctones no ambiente e sua utilização como recurso alimentar para Bryconamericus microcephalus (Miranda-Ribeiro, 1908). No período de maio de 2002 a fevereiro de 2003 foram realizadas as coletas para a quantificação do material alóctone importado, sendo paralelamente realizadas coletas de indivíduos da espécie para análise da dieta. A seletividade da espécie, no que se refere a dieta, foi estabelecida com base no tamanho e tipo das presas consumidas, considerando jovens e adultos separadamente. O índice de eletividade de Ivlev (IEI) foi aplicado com base na abundância dos itens disponíveis no ambiente e dos itens ingeridos pela espécie. Homoptera (IEI $=0,94)$, Coleoptera $(\mathrm{IEI}=0,57)$ e Diptera (IEI $=0,50$ ) apresentam preferência na dieta dos adultos e Hemiptera $(0,98)$, Diptera $(0,60)$ e Homoptera $(0,98)$ apresentam preferência na dieta dos jovens. Hymenoptera (IEI $=-0,15 ;-0,42)$ e Orthoptera (IEI $=-0,52 ;-1)$, apesar de estarem entre os mais abundantes no ambiente e serem consumidos pela espécie, não são itens preferenciais na dieta dos adultos e jovens, respectivamente, sugerindo que seu consumo é função da sua abundância. Os resultados apresentados indicam que jovens e adultos de B. microcephalus têm preferências distintas.

PALAVRAS CHAVE. Dieta; seletividade; floresta pluvial.

A decisão do predador de atacar ou não a presa é o resultado de uma atividade de procura e encontro, onde o tempo de procura e o respectivo gasto energético devem ser compensados através da energia contida na presa (GERKING 1994). Considerando que uma presa normalmente varia em abundância e disponibilidade, e que essas variáveis afetam o tempo de encontro, a "decisão" do predador deve levar em consideração a relação custo-benefício a fim de otimizar sua sobrevivência (WootTon 1998).
A maioria das espécies de peixes é forrageadora seletiva com preferência por presas específicas (Mittelbach \& Persson 1998). Por outro lado, a ingestão de um tipo de presa depende de sua abundância no ambiente mas, quando esta presa não está disponível, os predadores podem satisfazer suas necessidades nutricionais e energéticas com outro tipo de presa que, normalmente, será selecionada pela qualidade (PINNEGAR et al. 2003). Em riachos de mata fechada a vegetação marginal deter-

Revista Brasileira de Zoologia 23 (1): 218-222, março 2006 
mina uma grande redução na entrada de luz solar (STANFORD 1998) e, como conseqüência, a deriva de matéria orgânica importada supera os processos internos de produção (VANNOTE et al. 1980, Henry et al. 1994, Thorp 2002). Dessa forma, a grande disponibilidade de presas de origem terrestre é uma constante nesses ambientes (Henry et al 1994, Uieda \& KiKUChi 1995) e determina que esse recurso é de grande importância para as comunidades presentes no sistema (STANFord 1998). Dentre as comunidades que compõem os ambientes de riacho destaca-se a ictiofauna, com muitas espécies com dieta amplamente baseada nas presas alóctones.

Em riachos da Mata Atlântica, esse fato tem sido constantemente comprovado (Casatti \& Castro 1998, Mazzoni \& Rezende 2003, Rezende \& MAZzoni 2003) e, no caso da Ilha Grande, o córrego Andorinha se destaca por sua localização em extensa área com mata ciliar fechada. Estudos anteriores realizados nessa região apontaram o lambari Bryconamericus microcephalus (MirandaRibeiro, 1908) como uma das espécies de maior distribuição e abundância, com hábito alimentar insetívoro oportunista com predomínio de insetos alóctones (Rezende \& MAZzoni 2003).

Neste trabalho temos como objetivo determinar a relação entre o aporte e a disponibilidade de presas alóctones no Córrego Andorinha e sua utilização como recurso alimentar de B. microcephalus.

\section{MATERIAL E MÉTODOS}

A Ilha Grande $\left(23^{\circ} 11^{\prime} 11,7^{\prime \prime}\right.$ S e $\left.044^{\circ} 12^{\prime} 02,0^{\prime \prime} \mathrm{W}\right)$ está localizada na costa sul do Estado do Rio de Janeiro, entre as cidades de Mangaratiba e Angra dos Reis. O Parque Estadual da Ilha Grande compreende uma extensão de aproximadamente 30\% do total de 19.300 ha da ilha. Aproximadamente metade da área do Parque (47\%) é coberta por floresta densa, ombrófila, de Mata Atlântica (AlHo et al. 2002) e constitui áreas bem preservadas de mata primária e algumas áreas, mais baixas, de mata secundária.

O córrego Andorinha é um riacho de terceira ordem que se localiza na vertente continental da Ilha, percorre uma extensão de aproximadamente $10 \mathrm{Km}$, desde sua nascente até a desembocadura, na enseada dos Dois Rios e está, em parte, incluído dentro da área do parque. Ao longo do seu percurso, apresenta grande variação de relevo, intercalando áreas de corredeiras, rápidos e remansos e percurso subterrâneo em alguns trechos. A localidade de estudo, compreende um trecho de aproximadamente $80 \mathrm{~m}$ de extensão com cobertura vegetal bem preservada em aproximadamente $90 \%$ da localidade.

Durante o período de maio de 2002 á fevereiro de 2003 foram realizadas coletas bimestrais no Córrego Andorinha; em um trecho com extensão aproximada de 80 m que se caracteriza por uma área formada de corredeiras, intercaladas de poças marginais com substrato rochoso e/ou areno-lodoso. Nesse trecho foram realizadas coletas de indivíduos de Bryconamericus microcephalus através de arrastinhos (malha $0,5 \mathrm{~mm}$ ), rede de espera (malha $10 \mathrm{~mm}$ ), peneiras e puçás. As amostragens do material alóctone seguiram a metodologia descrita por ANGER-
MEIER \& KarR (1983) e adaptações de Henry et al. (1994) e UiedA \& KIKUCHI (1995). A cada coleta e localidade, quatro bandejas com dimensões de $0,4 \mathrm{~m} \mathrm{x} 0,2 \mathrm{~m}\left(0,8 \mathrm{~m}^{2}\right)$, pintadas de marrom e acopladas a flutuadores de isopor, foram parcialmente cheias com água do córrego, fixadas em pontos escolhidos aleatoriamente e expostas por $48 \mathrm{~h}$ (RezEnde \& MAZzoni 2005).

A matéria importada, obtida em cada bandeja, foi individualizada e conservada em álcool 70\%. A matéria animal encontrada foi contabilizada e identificada até o nível taxonômico de ordem, de acordo com Buzzi \& Miyazaki (1999) e consulta a especialistas. Posteriormente foi calculada a densidade de indivíduos coletados nas bandejas através da razão entre a área de exposição $\left(\mathrm{m}^{2}\right)$, o período de exposição (dia) e o número de indivíduos (ind) coletados: (ind. $\mathrm{m}^{2} \cdot \mathrm{dia}^{-1}$ ).

Os peixes coletados foram acondicionados em um isopor com gelo e levados ao laboratório onde foram dissecados e seus estômagos fixados em formal 5\% para posterior análise. Para análise do conteúdo estomacal foi utilizada a metodologia de freqüência numérica que consiste na contagem de indivíduos encontrados em cada estômago (Hyslop 1980, Zavala-CAMIN 1996). Exemplares testemunhos foram depositados na coleção ictiológica do Museu Nacional do Rio de Janeiro (MNRJ 28733).

Os exemplares jovens e adultos de B. microcephalus foram tratados separadamente a fim de se eliminar possíveis efeitos relacionados às variações ontogenéticas. A distinção dos indivíduos jovens e adultos foi definida de acordo com o tamanho da primeira maturação da espécie que ocorre no tamanho de 4,2 (MAzzoni \& Silva 2006). O tamanho dos exemplares jovens variou entre 2,5 e 4,2 enquanto que dos exemplares adultos o variou entre 4,3 e 7,6.

O tamanho das presas registradas no conteúdo estomacal dos exemplares analisados foi estimado para auxiliar na discussão sobre possíveis padrões de utilização das presas de diferentes tamanhos, por exemplares jovens e adultos. As presas consumidas tiveram o seu tamanho determinado a partir dos mesmos itens encontrados nos estômagos e nas bandejas. Como, em muitos casos, os organismos ingeridos encontravamse partidos, utilizou-se para a tomada das medidas os exemplares, da mesma espécie, das bandejas. As medidas foram realizadas com paquímetro digital, considerando-se, sempre, a maior extensão do indivíduo (comprimento ou largura).

A fim de se determinar a relação entre a disponibilidade e uso das presas foi utilizado o índice de Eletividade Ivlev, de acordo com o modelo a seguir: $\mathrm{IEI}=(r i-n i) /(r i+n i)$ onde: $(E i)$ índice de eletividade para a presa $\mathrm{i},(r i)$ percentagem da presa i na dieta, (ni) percentagem da presa i no ambiente. Os valores deste índice variam entre $-1,0$ e $+1,0$ sendo que os valores entre 0 e $+1,0$ indicam preferência e os valores entre -1,0 e 00 indicam rejeição (KREBS 1989).

\section{RESULTADOS}

Foram coletados 69 exemplares adultos e 12 exemplares jovens de $B$. microcephalus durante o período de estudo. Para a

Revista Brasileira de Zoologia 23 (1): 218-222, março 2006 
análise da dieta não foram consideradas variações sazonais, visto que, anteriormente havia sido detectada pouca variação no consumo de itens alóctones ao longo do ano.

Dentre os oito tipos de presas registrados nos experimentos de quantificação da matéria alóctone importada, observase que Hymenoptera e Orthoptera são os itens mais abundantes, representando $85,2 \%$ do total de itens animais importados (Tab. I).

Tabela I. Densidade média (ind $/ \mathrm{m}^{2}$ ), respectivos valores percentuais (\%) de potenciais presas e valores do Índice de Ivlev para exemplares jovens e adultos de Bryconamericus microcephalus aportadas no trecho estudado no córrego Andorinha no período de maio de 2003 a fevereiro de 2004.

\begin{tabular}{lccccc}
\hline \multirow{2}{*}{ Itens } & \multicolumn{2}{c}{ Densidade média } & & \multicolumn{2}{c}{ Índice de Ivlev } \\
\cline { 2 - 3 } \cline { 5 - 6 } & ${\text { Ind. }{ }^{2} \cdot \text { dia }^{-1}}^{1}$ & $\%$ & & Adulto & Jovem \\
\hline Hymenoptera & 80,3 & 70,7 & & $-0,14$ & $-0,42$ \\
Orthoptera & 16,5 & 14,5 & & $-0,51$ & $-1,00$ \\
Arachnida & 6,5 & 5,7 & & 0,04 & $-1,00$ \\
Coleoptera & 5,3 & 4,6 & & 0,58 & $-1,00$ \\
Diptera & 4,0 & 3,5 & & 0,51 & 0,60 \\
Odonata & 0,5 & 0,4 & & $-1,00$ & $-1,00$ \\
Hemiptera & 0,3 & 0,2 & & $-1,00$ & 0,98 \\
Homoptera & 0,3 & 0,2 & & 0,95 & 0,98 \\
\hline Total & 113,5 & \multicolumn{3}{c}{} \\
\hline
\end{tabular}

Contrapondo esses resultados com aqueles da dieta de jovens e adultos de B. microcephalus tem-se que, dentre as presas de origem alóctone, Hymenoptera, Homoptera, Hemiptera e Diptera são os únicos itens que compõem a dieta dos jovens (Fig. 1). Hymenoptera, Coleoptera e Diptera predominam na dieta dos adultos e perfazem $81,2 \%$ da dieta (Fig. 1).

Observa-se que, tanto para os jovens quanto para os adultos, a maior abundância dos itens consumidos é de presas com tamanho inferior a 3,33 $\mathrm{mm}$. Entre os jovens $71,4 \%$ da dieta se concentra nessa classe de tamanho e entre os adultos $64,1 \%$. Por outro lado, as presas de tamanho superior a $3,33 \mathrm{~mm}$ foram sempre consumidas pelos adultos, mesmo que em menor abundância, enquanto entre os jovens apenas homoptera $(6,80$ $\mathrm{mm}$ ) foi registrada na dieta (Fig. 1).

De acordo com os resultados do Índice de Ivlev (Tab. I) observa-se que Coleoptera, Diptera e Homoptera são as presas preferenciais dos exemplares adultos. Hymenoptera, apesar de ser o item mais abundante no ambiente é rejeitado, sugerindo que é um item consumido devido a sua grande disponibilidade no ambiente. Entre os exemplares jovens observa-se preferência por Hemiptera, Diptera e Homoptera e rejeição por todos as demais presas disponíveis. Sugere-se, assim, que tanto os jovens como os adultos são generalistas já que utilizam as presas de acordo com a sua disponibilidade no ambiente.

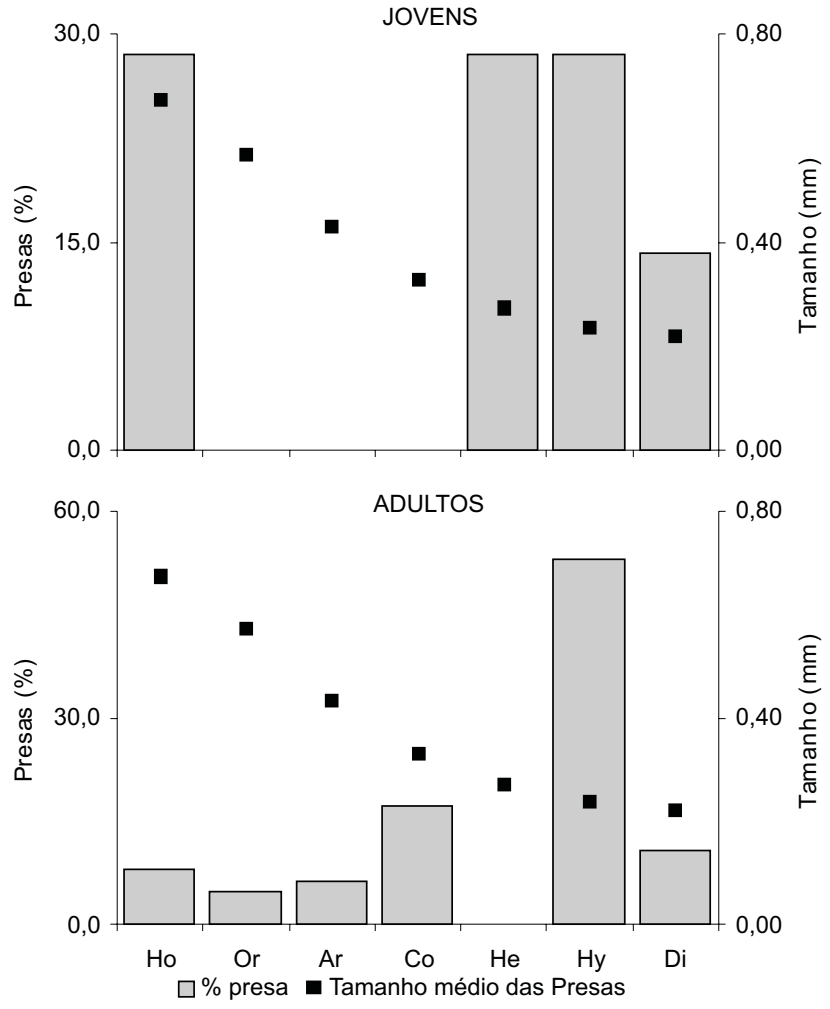

Figura 1. Análise da relação entre o tamanho médio e a abundância relativa das presas alóctones consumidas por Bryconamericus microcephalus do córrego Andorinha, Ilha Grande. (Ho) Homoptera, (Or) Orthoptera, (Ar) Arachnida, (Co) Coleoptera, (He) Hemiptera, (Hy) Hymenoptera, (D) Diptera.

\section{DISCUSSÃO}

Muitos predadores encontram suas presas na deriva, na vegetação marginal ou associadas a substratos que são utilizados tanto para a reprodução quanto para a alimentação (GILL \& HART 1999).

Dentre os itens que compõem a dieta de B. microcephalus os insetos alóctones corresponderam a uma importante parcela dos itens consumidos. De acordo com os nossos resultados, a abundância desses itens na dieta dos adultos é função da abundância dos mesmos no ambiente. Segundo Gotceitas \& Godin (1991) a disponibilidade de alimento é um importante fator que influencia o comportamento alimentar das espécies e, no caso de ambientes onde determinado alimento é escasso, a alteração na abundância dos itens consumidos é muito comum (Hojesjo et al. 1999, VeHANEN 2003).

Os resultados obtidos com o índice de Ivlev sugerem que B. microcephalus consome presas que, em teoria, seriam evitadas pela espécie; ou seja, os itens que estão disponíveis em grande quantidade no ambiente são consumidos, porém não de 
modo preferencial ou vice-versa. Este é o caso dos Hymenoptera que, apesar de terem sido consumidos correspondem a um item rejeitado $\left(\right.$ IEI $_{\text {adultos }}=-0,14$ e IEI $\left._{\text {jovens }}=-0,42\right)$. Da mesma forma, o item Homoptera apresenta reduzida disponibilidade no ambiente e o seu consumo é relativamente alto, indicando preferência na escolha desta presa. Esses resultados sugerem que $B$. microcephalus, apesar de apresentar um caráter oportunista na exploração dos recursos alimentares, exibe algum grau de seletividade alimentar.

A seletividade alimentar pode, além de relacionada com a qualidade do alimento, estar relacionada com o tamanho do mesmo. O tamanho da presa consumida por um predador pode variar de acordo com o tamanho do mesmo e, em uma mesma espécie, é comum que os jovens consumam presas menores enquanto os adultos consumam presas com tamanhos variados (Mittelbach \& Persson 1998). Da mesma forma, o tamanho mínimo de uma presa, para ser capturada, depende da capacidade de detecção do predador (PARMA \& Deriso 1990). Observamos que os indivíduos adultos de $B$. microcephalus consomem todos os tipos de presas disponíveis (exceto Odonata) independente do tamanho. Por outro lado, $71 \%$ da dieta dos jovens está baseada em presas de tamanho pequeno. Neste ponto, é importante ressaltar que o número amostral de jovens foi relativamente reduzido, como conseqüência das limitações metodológicas de coleta e da necessidade de não impor pressão predatória sobre a população de peixes de um ambiente isolado como a Ilha Grande. Assim sendo, os resultados aqui apresentados, para os jovens da espécie, devem ser tratados com cautela, mas são indicativos de que esse segmento da população de B. microcephalus possui restrições morfológicas que minimizam a utilização de presas maiores.

Concluímos que a quase totalidade das presas alóctones disponíveis no ambiente é utilizada por B. microcephalus como recurso alimentar. Mesmo as presas com baixos valores de preferência $(I E V<0)$ são consumidas, sugerindo uma dieta oportunista.

\section{AGRADECIMENTOS}

Este trabalho é parte da dissertação de mestrado da primeira autora (CFR) e foi financiado pelo projeto CNPq (APQ2002-479426/01-5). Agradecemos as bolsas individuais recebidas pelas autoras CFR - FAPERJ E-26/151.406/2003 e RM - CNPq 302628/2002-9 e a todos os membros do Laboratório de Ecologia de Peixes / UERJ pela ajuda nos trabalhos de campo.

\section{REFERÊNCIAS BIBLIOGRÁFICAS}

Alho, C.J.R.; M. Schneider. \& L.A. Vasconcellos. 2002. Degree of the threat to the biological diversity in the Ilha Grande state park (RJ) and the guidelines for conservation. Brazilian Journal of Biology, São Carlos, 62 (3): 375-385.

Angermeier, P.L. \& J.R. Karr. 1983. Fish communities along environmental gradientes in a system of tropical streams.
Environmental Biology of fishes, Dordrecht, 9 (2): 117135.

Buzzi, Z.J. \& D.R. Miyazaki. 1999. Entomologia didática. Curitiba, Editora Universidade Federal do Paraná, 308p.

Casatti, L. \& R.M.C. Castro. 1998. A fish community of the São Francisco River headwater riffles, southeastern Brazil. Ichthyological Exploration of Freshwaters, Munchen, 9: 229-242.

Gerking, S.D. 1994. Feeding ecology of fish. San Diego, Academic Press, 414p.

Gill, A.B. \& P.J.B. HarT. 1999. Dynamic changes in prey choice by stickleback during simultaneous encounter with large prey. Journal of Fish Biology, London, 55: 1317-1327.

Gotceitas, V. \& J.G.J. Godin. 1991. Foraging under the risk of predation in juvenile Atlantic salmon (Salmo salar L.): effects of social status and hunger. Behavioral Ecology and Sociobiology, New York, 29: 255-261.

Henry, R.; V.S. Uieda; A.A. Afonso \& R.M. KikUChI. 1994. Input of allochthnous matter and structure of fauna in Brazilian headstream. Verhandlungen der Internationale Vereinigung fur Theoretische und Angewandte Limnologie, Stuttgart, 25 (3): 1867-1869.

Hyslop, E.J. 1980. Stomach contents analysis - a review of methods and their application. Journal of Fish Biology, London, 17: 411-429.

Hojesjo, J.; J. Johnsson \& M. Axelsson. 1999. Behavioural and heart rate responses to food limitation and predation risk: an experimental study on rainbow trout. Journal of Fish Biology, London, 55: 1009-1019.

Krebs, C.J. 1989. Ecological Methodology. New York, Harper Collins Publishers, XII+654p.

Mazzoni, R. \& C.F. Rezende. 2003. Seasonal diet shift in a Tetragonopterinae (Osteichthyes, Characidea) from Ubatiba river, RJ, Brazil. Brazilian Journal of Biology, São Carlos, 63 (1): 69-74.

Mazzoni, R. \& A.P.F. Silva. 2006. Aspectos da história de vida de Bryconamericus microcephalus (Miranda Ribeiro) (Characiformes, Characidae) de um riacho costeiro de Mata Atlântica, Ilha Grande, Rio de Janeiro, Brasil. Revista Brasileira de Zoologia, Curitiba, 23 (1): 228-233.

Mittelbach, G.G. \& L. Persson. 1998. The ontogeny of piscivory and its ecological consequences. Canadian Journal of Fisheries and Aquatic Sciences, Ottawa, 55: 1454-1465.

Parma, A.M. \& R.B. Deriso. 1990. Dynamics of age and size composition in a population subject to size-selective mortality: effects of phenotypic variability in growth. Canadian Journal of Fisheries and Aquatic Sciences, Ottawa, 47: 274-289.

Pinnergar, J.K.; V.M. Trenkel; A.N. Tidd; W.A. Dawson \& M.H. DU Buтr. 2003. Does diet in Celtic Sea fishes reflect prey availability?. Journal of Fish Biology, London, 63 (A): 197-212.

Rezende, C.F. \& R. Mazzoni. 2003. Aspectos da alimentação de Bryconamericus microcephalus (Characiformes, Tetragonopte- 
rinae) no Córrego Andorinha, Ilha Grande - RJ. Biota Neotropica, Campinas, 3 (1): 1-6.

Rezende, C.F. \& R. Mazzoni. 2005. Seasonal variation in the input of allochthonous matter in an Atlantic Rain Forest stream, Ilha Grande-RJ. Acta Limnologica Brasiliensia, São Paulo, 17 (2): 167-175.

STANFORD, J.A. 1998. Rivers in the landscape: introduction to the special issue on riparian and groundwater ecology. Freswater Biology, Oxford, 40: 402-406.

Thorp, J.H. 2002. Dominance of autochthonous autotrophic carbon in food webs of heterotrophic rivers. Oikos, Copenhagen, 93 (3): 543-550.

UiedA, V.S. \& R.M. KiKUCHI. 1995. Entrada de material alóctone (detritos vegetais e invertebrados terrestres) num pequeno curso de água corrente na costa de Botucatu, São Paulo. Acta Limnologica Brasiliensia, São Paulo, 7: 105-114.

Vannote, R.L.; G.W. Minshall; K.W. Cummins; J.R. Sedell \& C.E. CusHING. 1980. The river continuum concept. Canadian Journal of Fisheries and Aquatic Sciences, Ottawa, 37: 130-137.

Vehanen, T. 2003. Adaptive flexibility in the behavior of juvenile Atlantic salmon: short-term responses to food availability and threat from predation. Journal of Fish Biology, London, 63: 1034-1043.

ZaVAla-CAMIN, L.A. 1996. Introdução ao estudo sobre alimentação natural em peixes. Maringá, Editora Universidade Estadual de Maringá, 129p.

Wootton, R.J. 1998. Ecology of teleost fishes. Dordrecht, Academic Publishers, 392p.

Recebido em 06.V.2005; aceito em 22.XI.2005. 